\title{
IDEAL THEORY ON OPEN RIEMANN SURFACES
}

\author{
O. F. G. SCHILLING
}

Introduction. The theorems of the classical ideal theory in fields of algebraic numbers hold in rings of analytic functions on compact Riemann surfaces. The surfaces admitted in our discussion are closely related to algebraic surfaces; we deal either with compact surfaces from which a finite number of points are omitted or, more generally, with surfaces determined by an algebroid function. The local aspects of the resulting ideal theory are the same as those found in the theory of algebraic functions. However, the ideal theory in the large is quite different. We now cannot count on the simplifications which are implied by the ascending chain condition for ideals. The recent theories of functions on topological spaces provide the necessary tools for a simple theory in the large. We shall show that the topologization of rings of entire functions by means of the topology of the underlying surface furnishes a fruitful method. Thus, the closed maximal ideals will correspond to the points of the given surfaces. Finally, to quote another result, all closed ideals are principal.

1. Some basic definitions. Suppose that $\Sigma$ is the complex number sphere whose points at finite distance $p=p_{a}$ are in 1-1 correspondence with the elements $a$ of the complex number field $C$. We assume that each point $p$ of $\Sigma$, the point at infinity included, has conformal neighborhoods. In general let $t_{p}$ be a local uniformizing variable at $p$, that is, a function which affords the mapping of a conformal neighborhood of $p$ in the unit circle $\left|t_{p}\right|<1$. We associate with each point $p$ a model $\Sigma_{p}$ of $\Sigma$ and consider functions $f(z)$ on $\Sigma$ with values in $\left\{\Sigma_{p}\right\}$, in other words, $f(p) \in \Sigma_{p}$ for $p \in \Sigma$. As usual we say that a function $f(z)$ is meromorphic on $\Sigma$ if there exists for each point $p \in \Sigma$ a convergent expansion

$$
f(z)=\sum_{i=m}^{\infty} c_{p, i} i_{p}^{i}, \quad c_{p, i} \in C,
$$

where the first nonvanishing coefficient $c_{p, m}$ has a finite integral subscript. Then all meromorphic functions on $\Sigma$ form a field $F(\Sigma)$ which contains $C[2,17] .^{1}$

Next we associate to each function $f(z)$ the integer $m=V_{p} f(z)$ and

Lectures delivered before the Chicago meeting of the Society on April 26-27, 1946, by invitation of the Committee to Select Hour Speakers for Western Sectional Meetings; received by the editors July 26, 1946.

1 The numbers in brackets refer to the list of references. 
define $V_{p} 0=\infty$. The algebra of power series implies that the "valuation" $V_{p}$ has the following properties

$$
\begin{gathered}
V_{p}\left(t_{p}\right)=1, \quad V_{p}(f+g) \geqq \min \left(V_{p} f, V_{p} g\right), \\
V_{p}(f g)=V_{p}(f)+V_{p}(g), \quad V_{p} c=0
\end{gathered}
$$

for each nonzero complex number $[6,12,16]$. We note that $V_{p}$ measures the order of the zero or pole of $f$ at the point $p$. Furthermore the value $V_{p} f$ does not depend on the special choice of the variable $t_{p}$, that is, if $t_{p}^{\prime}=\sum_{i=1}^{\infty} d_{i} t_{p}^{t}, d_{1} \neq 0, d_{i} \in C$, is analytic in a sufficiently small neighborhood of $t_{p}=0$, and $f(z)$ is expanded with respect to $t_{p}^{\prime}$ as $\sum_{i=m}^{\infty}, c_{p, i}^{\prime} t_{p}^{\prime s}$, then $m=m^{\prime}$. We mention in this connection that the function theoretic value $f(p)$ of $f(z)$ at $p$ is:

(i) the coefficient $c_{p, 0}$ of (1) if $V_{p} f(z)=0$,

(ii) the element 0 if $V_{p} f(z)>0$, and

(iii) the element $\infty$ of $\Sigma_{p}$ if $V_{p} f(z)<0$, or if $f(p)^{-1}=0$.

Then, for the functions $f_{1}, f_{2}$, with $f_{1}(p), f_{2}(p) \neq \infty$,

$$
\left(f_{1}+f_{2}\right)(p)=f_{1}(p)+f_{2}(p), \quad\left(f_{1} f_{2}\right)(p)=f_{1}(p) f_{2}(p) .
$$

Thus the functions $f$ of $F(\Sigma)$ which are holomorphic at $p$ form a ring $R_{p}$, the so-called valuation ring of $p$ in $F(\Sigma)$. This ring contains a single prime ideal $M_{p}^{*}$ which consists of all functions which vanish at $p$. In particular, the homomorphism $R_{p} \rightarrow R_{p} / M_{p}^{*}=\phi_{p}\left(R_{p}\right)$ is the arithmetic description of the function theoretic value, $f(p)=\phi_{p}(f(z))$.

We recall next the following consequences of the compactness of $\Sigma:$

(4) If $f(p) \neq 0, \infty$ for all $p \notin \Sigma$ then $f$ is a nonzero complex number,

(5) If $f \notin C$, then $f(p)$ is 0 or $\infty$ for only a finite set of points of $\Sigma$, that is, a meromorphic function has a finite number of zeros and poles.

After these preparations it is a simple matter to construct the field $F(\Sigma)$. We first note that $z-a, a \in C$, is a meromorphic function with $V_{p}(z-a)=1$ with $p=p_{a}, V_{p}(z-a)=-1$ with $p=p_{\infty}, V_{p}(z-a)$ $=0$ for all other points $p$. Suppose that $f(z) \in F(\Sigma)$ has its poles and zeros at the points $p_{a_{1}}, \cdots, p_{a_{r}}$ where repetitions of the points and the point $p_{\infty}$ are permitted. Then the function $\prod_{i=1}^{r}\left(z-a_{i}\right)=f^{*}(z)$ has the same poles and zeros as $f(z)$. Using the second of rules (2) and (4) we find that $f(z) / f^{*}(z)$ is a nonzero constant $c$ of $C$. Thus

$$
f(z)=c f^{*}(z),
$$

or $F(\Sigma)$ is the field of all rational functions of the complex variable $z$.

2. The field of meromorphic functions. We omit now from $\Sigma$ the 
point $p_{\infty}$ and obtain thus the open complex number plane $\Sigma^{\prime}$ whose points are in 1-1 correspondence with the numbers of $C$. The space $\Sigma^{\prime}$ is locally compact and the sum of the compact regions $|z| \leqq n$, $n \rightarrow \infty$. We define a meromorphic function $f(z)$ on $\Sigma^{\prime}$ by omitting in the definition of $F(\Sigma)$ the condition of rationality at $p_{\infty}$. Then the field of meromorphic functions $F\left(\Sigma^{\prime}\right)$ contains $F(\Sigma)$ as proper subset. Statement (5) is now replaced by:

(7) A meromorphic function $f(z)$ has only a finite number of zeros and poles in a small neighborhood of each point $p \in \Sigma^{\prime}$.

The field $F\left(\Sigma^{\prime}\right)$ contains as a subring $O\left(\Sigma^{\prime}\right)$ the integral domain of all entire functions $f(z)$ with $V_{p} f(z) \geqq 0$ for all $p \in \Sigma^{\prime}$. Suppose that $f(z)$ is not a unit, that is, $f(z)$ has zeros $a_{j}$. Then the set of zeros $\left\{a_{j}\right\}$ is countable and (7) implies $\left|a_{j}\right| \rightarrow \infty$ as $j \rightarrow \infty$; moreover, only a finite number of $a_{j}$ 's have the same absolute value. The construction leading to (6) must now be replaced by Weierstrass' theorem [3]. We pick integers $m_{j}$ such that sum $\sum_{j=1}^{\infty} z^{m_{j}} / a_{j}^{m_{j}+1}, a_{j} \neq 0$, is absolutely convergent for all $z$. Then we define $q_{j}(z)=\sum_{k=1}^{m_{j}}(1 / k)^{k}\left(z / a_{j}\right)^{k}$ and set $f^{*}(z)=\prod_{j}\left(1-z / a_{j}\right) e^{q_{j}(z)}$. The product $f^{*}(z)$ is absolutely convergent for all $z$ and uniformly convergent in any finite region of $\Sigma^{\prime}$. Therefore $f^{*}(z) \in F\left(\Sigma^{\prime}\right)$. Suppose $V_{p_{0}} f(z)=m$. Then $f(z) /\left(z^{m} f^{*}(z)\right)$ has no zeros and poles on $\Sigma^{\prime}$. Hence it is a unit function $e^{o(z)}$ of $O\left(\Sigma^{\prime}\right)$ and

$$
f(z)=e^{o(z)} z^{m} \prod_{j}\left(1-z / a_{j}\right) e^{q_{j}(z)} .
$$

Conversely there always exists an entire function (8) with prescribed zeros. Moreover, an arbitrary function of $F\left(\Sigma^{\prime}\right)$ is always a quotient of entire functions, as follows by constructing entire functions according to (8) separately for its zeros and poles. Consequently $F\left(\Sigma^{\prime}\right)$ is the quotient field of $O\left(\Sigma^{\prime}\right)$.

3. The ring of entire functions. We note from the theory of polynomials that the polynomial ring $C[z]$ is equal to the intersection of the valuation rings $R_{p}$ for all $p \in \Sigma^{\prime}$. The definition of a function $f(z) \in F\left(\Sigma^{\prime}\right)$ implies that each point $p \in \Sigma^{\prime}$ determines again a valuation $V_{p}$ of $F\left(\Sigma^{\prime}\right)$ with a corresponding valuation ring $R_{p}^{\prime}$ in $F\left(\Sigma^{\prime}\right)$, for only local properties of functions are used. Moreover, the definition of an entire function shows that

$$
O\left(\Sigma^{\prime}\right)=\bigcap_{p} R_{p}^{\prime} \supset C[z] .
$$

Furthermore, each ring $R_{p}^{\prime}$ has a unique prime ideal $M_{p}^{\prime}$ consisting of all functions vanishing at $p$ such that, for $p=p_{a}, M_{p}^{\prime} \cap O\left(\Sigma^{\prime}\right)$ $=M_{p}=O\left(\Sigma^{\prime}\right)(z-a)$. Thus, each point $p \in \Sigma^{\prime}$ determines uniquely the 
prime ideal $M_{p} \subset O\left(\Sigma^{\prime}\right)$ which consists of all entire functions vanishing at $p$.

We next define an ideal $A$ of $O\left(\Sigma^{\prime}\right)$ to be an additive subgroup of $O\left(\Sigma^{\prime}\right)$ which admits the elements of $O\left(\Sigma^{\prime}\right)$ for multipliers. It has been noticed before that the (trivial) ideal theory of $C[z]$ has no direct analogue in $O\left(\Sigma^{\prime}\right)$ [7]. The ideal theory of $C[z]$ and its finite algebraic extensions has two characteristic properties. First there are no properly ascending chains of ideals and secondly there are no other prime ideals but the ones corresponding to points of the associated algebraic curve or Riemann surface. The ring $O\left(\Sigma^{\prime}\right)$ presents an entirely different arithmetic structure. Consider the entire function $\sin (\pi z)$ with the zeros $\pm n$ and set $f_{1}=\sin (\pi z), f_{2}=\sin (\pi z) / z, f_{3}$ $=\sin (\pi z) / z\left(z^{2}-1^{2}\right), \cdots, f_{n+2} \sin (\pi z) / z\left(z^{2}-1^{2}\right) \cdots\left(z^{2}-n^{2}\right)$. Then $f_{n+2}$ has the zeros $\pm(n+1), \cdots$ and $f_{1}=f_{n+2} z\left(z^{2}-1^{2}\right) \cdots\left(z^{2}-n^{2}\right)$. Then $O\left(\Sigma^{\prime}\right) f_{1} \subset O\left(\Sigma^{\prime}\right) f_{2} \subset \cdots$ is a properly ascending infinite chain of divisors. Moreover, admitting only finite sums, the join $U_{i} O\left(\Sigma^{\prime}\right) f_{i}$ $=\left(f_{1}, f_{2}, \cdots\right)=A$ is not the unit ideal $O\left(\Sigma^{\prime}\right)$, for otherwise $1=\sum_{i=1}^{N} g_{i} f_{i}$ with entire functions $g_{i}$. Such an equation cannot hold since the sum has infinitely many zeros.

The following remark indicates that a change of the definition of an ideal may be useful. Recalling the expansion $\pi / \sin (\pi z)=1 / z$ $+2 \sum_{n=1}^{\infty}\left[(-1)^{n} z\right] /\left(z^{2}-n^{2}\right)$ we obtain $1=(1 / \pi)\left[f_{1}-2 z^{2} f_{2}+2 z^{2}\left(z^{2}-1^{2}\right) f_{3}\right.$ $+\cdots]$. Thus 1 is an infinite convergent sum of elements in $A$.

There are three obvious devices which may be investigated in order to develop an ideal theory in $O\left(\Sigma^{\prime}\right)$ such that the resulting theory resembles the ideal theory of fields of algebraic functions or algebraic numbers. The first method provides a recasting of the definition of an ideal, the second utilizes the technique of $v$-ideals and quasi equality, and the third requires a restriction to ideals with a finite basis. The last approach was used by Helmer [7]. We shall use in the sequel the first and second methods.

We now introduce a topology in $O\left(\Sigma^{\prime}\right)$ in order to carry out the first method.

Definition 1. Suppose that $\left\{f_{n}\right\}$ is an infinite sequence of entire functions. We define $f_{n} \rightarrow f$ as $n \rightarrow \infty$ to mean uniform convergence in any bounded region of $\Sigma^{\prime}$.

Definition 2. An ideal $A$ of $F\left(\Sigma^{\prime}\right)$ with respect to $O\left(\Sigma^{\prime}\right)$ is called closed, if:

(i) $A$ is closed under addition and under multiplication by elements of $O\left(\Sigma^{\prime}\right)$, that is, $A$ is an ideal in the algebraic sense;

(ii) There exists a nonzero integral function $f$ such that $f A \leqq O\left(\Sigma^{\prime}\right)$; 
(iii) $A$ is closed in the topology of Definition 1.

We note that (iii) implies that $A$ contains all convergent infinite sums $\sum_{i=1}^{\infty} a_{i}$ for which $a_{i} \in A$.

Let

$$
V A=\left\{\min _{a \in A} V_{p} a=V_{p} A, \text { all } p \in \Sigma^{\prime}\right\} .
$$

This vector $U A$ has at most an enumerable infinitude of components $V_{p} A$ distinct from 0 since an element $a \in A$ has at most a countable set of zeros in $\Sigma^{\prime}$.

LEMmA 1. If $A$ is closed and $U A=\{0\}$, then $A=O\left(\Sigma^{\prime}\right)$.

Proof. It suffices to show that $A$ contains an entire function without zeros. Let $g \in A$ and suppose $a_{1}, a_{2}, \cdots, a_{n}, \cdots$ are the zeros of $g$. Since $U A=\{0\}$ there exists for each $a_{n}$ a function $f_{n} \in A$ with $V_{n} f_{n}=0$ or $f_{n}\left(a_{n}\right) \neq 0$, where $V_{n}$ is the valuation for $a_{n}$. Applying (8) we can exhibit a function $f \in O\left(\Sigma^{\prime}\right)$ which has simple zeros at the points $a_{n}$. Suppose that $\left|\left(f f_{n}\right) /\left(z-a_{n}\right)\right| \leqq M_{n}$ on $|z| \leqq n$. Then the sum $\sum_{n=1}^{\infty}\left(f f_{n}\right) /\left[\left(z-a_{n}\right) 2^{n} M_{r}\right]=h$ exists and represents an element of $O\left(\Sigma^{\prime}\right)$. By construction $h\left(a_{n}\right) \neq 0$. Consequently $g$ and $h$ are entire functions without common zeros. Hence there exist integral functions $k$ and $l$ such that $g k+h l=1$ (see [7, pp. 351-352]). Therefore $1 \in A$ or $A=O\left(\Sigma^{\prime}\right)$. lemma.

Using a repeated diagonal process we can prove the following

Lemma 2. Let $A, B$ be two closed ideals and define the product $A \bigcirc B$ as the totality of all convergent sums $\sum_{n=1}^{\infty} a_{n} b_{n}, a_{n} \in A, b_{n} \in B$. Then $A \bigcirc B$ is closed.

Lemma 3. All closed ideals $A$ are principal.

Proof. Weierstrass' theorem (8) together with (10) implies the existence of an element $a \in O\left(\Sigma^{\prime}\right)$ with $V_{p} a=V_{p} A$ for all $p \in \Sigma^{\prime}$. Then the set of all quotients $b / a, b \in A$, is an ideal $B$ in $O\left(\Sigma^{\prime}\right)$. The ideal $B$ is closed for $b_{n} / a \rightarrow g, b_{n} \in B$, implies $g=h / a$ with $h \in O\left(\Sigma^{\prime}\right)$; but $b_{n} \rightarrow a g$ and therefore $a g \in A$ since $A$ is closed. By construction $\mathcal{V} B=\{0\}$ and therefore, by Lemma $1, B=O\left(\Sigma^{\prime}\right)$ or $A=a O\left(\Sigma^{\prime}\right)$.

In order to describe the ideal theory of the closed ideals of $F\left(\Sigma^{\prime}\right)$ with respect to $O\left(\Sigma^{\prime}\right)$ we introduce the following vector group $U$ in which addition is defined by addition of the components [10]. We consider vectors of integers $\left\{m_{p}, p \in \Sigma^{\prime}\right\}$ where at most a denumerable infinitude of components $m_{p_{a_{n}}}$ do not vanish and where $\left|a_{n}\right| \rightarrow \infty$ 
as $n \rightarrow \infty$. The mapping $A \rightarrow \cup A \in U$ maps the group of closed ideals $\{A\}$ (with the multiplication of Lemma 2) into $U$ in such fashion that $V(A O B)=V(A)+V(B)$ and $V\left(O\left(\Sigma^{\prime}\right)\right)=\{0\}$. Since all closed ideals are principal we have trivially $\mho A^{-1}=-V A$ where $A^{-1}$ is the closed ideal of all $b \in F\left(\Sigma^{\prime}\right)$ with $b A \subseteq O\left(\Sigma^{\prime}\right)$. Conversely, Weierstrass' theorem (8) implies that each vector $\left\{m_{p}, p \in \Sigma^{\prime}\right\}$ of $U$ is the vector $\bigcup A$ of a closed ideal $A$. Observe that $A=O\left(\Sigma^{\prime}\right) a$ for $a \in F\left(\Sigma^{\prime}\right)$ with $V_{p} a=m_{p}$ for all $p \in \Sigma^{\prime}$. The ideal $A$ is unique since $V A_{1}=V A$ for any other ideal $A_{1}$ with $U A_{1}=\left\{m_{p}, p \in \Sigma^{\prime}\right\}$ and since $A$ is the totality of all functions $c$ with $V_{p} c \geqq m_{p}$ for all $p \in \Sigma^{\prime}$. We have proved:

Theorem 1. The closed ideals $A$ of $F\left(\Sigma^{\prime}\right)$ with respect to $O\left(\Sigma^{\prime}\right)$ form a multiplicative group which is isomorphic to the vector group $U$.

We mention in passing that we may consider infinite products of ideals, provided obvious assumptions are made on the distribution of the zeros and poles.

The second method which utilizes the theory of quasi equality is closely related to the first, as the following observations show.

Definition 3. Two ideals, $A_{1}, A_{2}$, of $F\left(\Sigma^{\prime}\right)$ with respect to $O\left(\Sigma^{\prime}\right)$ are called quasi equal, $A_{1} \sim A_{2}$, if $\mho A_{1}=V A_{2}[10]$.

We now consider the classes of quasi equal ideals $[A]$ and define $[A][B]=[A B]$. This definition will be useful if we can show that quasi equality is an equivalence relation. For the proof we note:

$$
V(A)=\{0\} \text { if and only if } A^{-1}=O\left(\Sigma^{\prime}\right) .
$$

We have $V A^{-1}=\{0\}$ and thus $A^{-1}=O\left(\Sigma^{\prime}\right)$ since $A^{-1}$ is closed. For the converse we observe that $V A=-V A^{-1}=\{0\}$.

$$
(A B)^{-1}=A^{-1} \text { for any ideal } B \text { with } \cup B=\{0\} \text {. }
$$

Note that $A B \subseteq A$ implies $(A B)^{-1} \supseteq A^{-1}$. For the converse let $c \in(A B)^{-1}$, then $O\left(\Sigma^{\prime}\right) \supseteq c(A B)=(c A) B$. Consequently $c A \subseteq B^{-1}$. Therefore, by (11), $c A \subseteq O\left(\Sigma^{\prime}\right)$ or $c \in A^{-1}$. Hence $(A B)^{-1} \subseteq A^{-1}$.

(13) $A \sim B$ if and only if $A^{-1}=B^{-1}$, and there exist ideals $C_{1}, C_{2}$ with $V C_{1}=V C_{2}=\{0\}$ such that $A C_{1}=B C_{2}$.

Consider $A A^{-1} B=A\left(A^{-1} B\right)=\left(A A^{-1}\right) B$ and put $A^{-1} B=C_{1}$, $A A^{-1}=C_{2}$. Then by (12), $A^{-1}=\left(A C_{1}\right)^{-1}=\left(B C_{2}\right)^{-1}=B^{-1}$, for $V C_{1}=V C_{2}=\{0\}$ The converse is obvious.

We can therefore assert that the group of classes of quasi equal ideals is isomorphic to the vector group $U$ where the isomorphism is established by the mapping $[A] \rightarrow \bigcup A$. This statement can be interpreted further. 
Lemma 4. Let $\bar{A}$ be the closure of the ideal $A$, in the sense of the Definition 1, then $V \bar{A}=V A$.

For the proof note that $\bar{A} \supseteq A$ implies $V_{p} \bar{A} \leqq V_{p} A$ for all $p \in \Sigma^{\prime}$. For the converse we may assume without loss of generality that $A \subseteq O\left(\Sigma^{\prime}\right)$. Let $a=\lim _{n \rightarrow \infty} a_{n}, a_{n} \in A$, be an arbitrary element of $\bar{A}$. Suppose that $V_{p} A=m>0, b \in C$ with $p=p_{b}$. We consider the sequence $\left\{b_{r}=a_{n}(z-b)^{m-1}\right\}$ with the limit $f=a(z-b)^{m-1}$. We now pick a positive $\epsilon$ so small that $\left|b_{n}-f\right|<\epsilon / 2$ in a sufficiently small neighborhood of $p_{b}$ in $\Sigma^{\prime}$. Since $f$ is analytic we have $|f-f(b)|<\epsilon / 2$ and consequently $\left|b_{n}-f(b)\right|<\epsilon$. If $f(b)$ were distinct from zero, then $\epsilon$ can be chosen such that $\epsilon<|| b_{n}|-| f(b)||<\left|b_{n}-f(b)\right|$. Hence, by contradiction, $f(b)=0$ and thus $V_{p} a \geqq V_{p} A$. Consequently $V_{p} \bar{A} \geqq V_{p} A$ and therefore $V \bar{A}=V A$. Consequently:

(14) All ideals in [A] have the same closure $\bar{A}$, and $\bar{A}$ is the largest ideal in $[A]$.

Thus Theorem 1 states that the group theoretical structure of the set of all class of quasi equal ideals is the same as the structure of the group of all closed ideals.

4. The prime ideals of $O\left(\Sigma^{\prime}\right)$. We noticed in $\$ 2$ that each point $p$ of $\Sigma^{\prime}$ gives rise to a valuation of $F\left(\Sigma^{\prime}\right)$; moreover, $p$ determines uniquely a maximal prime ideal $M_{p}$ of $O\left(\Sigma^{\prime}\right)$. The results of $\$ 3 \mathrm{imply}$ that the prime ideal $M_{p}$ is closed since it consists of all entire functions which vanish at $p$. We now show that $O\left(\Sigma^{\prime}\right)$ contains infinitely many nonclosed prime ideals $M$. The example of $\S 3, A=(\sin (\pi z)$, $\sin (\pi z) / z, \cdots) \subset O\left(\Sigma^{\prime}\right)$ proves the existence of a nonclosed ideal with $U(A)=\{0\}$. Applying Zorn's lemma we can prove the existence of a prime ideal $M \supset A, M \neq O\left(\Sigma^{\prime}\right)$ with $U M=\{0\}$. There are infinitely many such prime ideals. Pick two sequences of complex numbers $a_{n}, b_{n}$ with $\left|a_{n}\right|,\left|b_{n}\right| \rightarrow \infty$ which are distinct. By (8) there exist functions $f_{n}, g_{n}$ with zeros at $a_{n}, a_{n+1}, \cdots$ and $b_{n}, b_{n+1}, \cdots$, respectively. Let $B=\left(f_{1}, f_{2}, \cdots\right)$ and $C=\left(g_{1}, g_{2}, \cdots\right)$, then $U B=V C$ $=\{0\}$. We assert that $B$ and $C$ determine distinct maximal prime ideal divisors. Suppose $B \subset M_{1}, C \subset M_{2}$ and $M_{1}=M_{2}$. Then $f_{1}$, $g_{1} \in M_{1}=M_{2}$. Hence there exist entire functions $a, b$ such that $f_{1} a+g_{1} b=1 \in M_{1}=M_{2}=O\left(\Sigma^{\prime}\right)$ because $f_{1}, g_{1}$ have distinct zeros. Therefore $M_{1} \neq M_{2}$.

Lemma 5. All prime ideals of $O\left(\Sigma^{\prime}\right)$ are maximal.

Proof. If a prime ideal $M \subset O\left(\Sigma^{\prime}\right)$ were not maximal, there would exist a function $f \in O\left(\Sigma^{\prime}\right)$ for which $(M, f) \subset O\left(\Sigma^{\prime}\right)$. Let $g \in M$ and suppose that $\left\{a_{n}\right\}$ is the set of common zeros of $f$ and $g$. By (8) 
there exists a function $h \in O\left(\Sigma^{\prime}\right)$ with precisely the zeros $\left\{a_{n}\right\}$. We write $g=h m$. Hence either $h \in M$ or $m \in M$. But $m$ does not lie in $M$ for $m$ and $f$ have no common zeros and thus generate $O\left(\Sigma^{\prime}\right)=(m, f)$, whence $(M, f)=O\left(\Sigma^{\prime}\right)$, contrary to hypothesis. Therefore $h \in M$ and $f \in M$ since $f$ is a multiple of $h$. Thus $M$ is maximal.

This lemma implies that each closed prime ideal $M$ of $O\left(\Sigma^{\prime}\right)$ belongs to a point, that is, that there exists a unique $p$ with $M=M_{p}$. Note that Lemma 1 implies $V_{p} M \neq 0$ for some $p$. Hence $M \subseteq M_{p}$ and thus $M=M_{p}$ by maximality. We note that $O\left(\Sigma^{\prime}\right) / M_{p} \cong C$.

The nonclosed prime ideals $M$ can be characterized algebraically as follows.

Lemma 6. A maximal ideal $M$ of $O\left(\Sigma^{\prime}\right)$ is nonclosed if and only if $O\left(\Sigma^{\prime}\right) / M$ is a proper extension of the complex number field $C$.

Proof. For a prime ideal $M$ certainly $M \cap C[z] \neq C[z]$, since $M$ would otherwise contain a nonzero constant and be equal to $O\left(\Sigma^{\prime}\right)$. Consequently either $M \cap C[z] \neq 0, C-0 \notin M \cap C[z]$, or $M \cap C[z]=0$. In the first case $M \cap C[z]=(z-a)$ for some $a \in C$ because $M$ is prime. Consequently $O\left(\Sigma^{\prime}\right)(z-a)=M_{p_{a}} \subseteq M$ and $M=M_{p_{a}}$ is closed. Hence $M \cap C[z]=0$ for a nonclosed prime ideal. But then $O\left(\Sigma^{\prime}\right) / M$ contains $C[z] / 0 \cong C[z]$ and therefore $O\left(\Sigma^{\prime}\right) / M \supset C$. Conversely $O\left(\Sigma^{\prime}\right) / M$ $\supset C$ does not hold for closed prime ideals, hence $M$ is not closed.

We consider next valuations $V$ of $F\left(\Sigma^{\prime}\right)$ whose valuation rings contain $O\left(\Sigma^{\prime}\right)$. Assume that $V$ does not arise from a point $p \in \Sigma^{\prime}$. Then the prime ideal $P$ of the valuation ring of $V$ in $F\left(\Sigma^{\prime}\right)$ necessarily meets $O\left(\Sigma^{\prime}\right)$ in a nonclosed prime ideal $M=P \cap O\left(\Sigma^{\prime}\right)$. We note that then $V M=\{0\}$, for otherwise there would exist a point $p$ with $V_{p} M \neq 0$. Then $M \subseteq M_{p}$ or $V=V_{p}$ contrary to assumption. We assert:

LEMma 7. There exists no valuation $V$ of rank one such that a nonclosed prime ideal $M$ of $O\left(\Sigma^{\prime}\right)$ is equal to $P \cap O\left(\Sigma^{\prime}\right)$ where $P$ is the prime ideal of $V$.

Proof. We have $U\left[P \cap O\left(\Sigma^{\prime}\right)\right]=\{0\}$. Let $f \in P \cap O\left(\Sigma^{\prime}\right)$, then $f$ must have infinitely many zeros; for if $f$ were a unit then $P \cap O\left(\Sigma^{\prime}\right)$ $=O\left(\Sigma^{\prime}\right)$, and if $f$ had a finite number of zeros then $V$ would be some $V_{p}$. Let $a_{1}, a_{2}, \cdots$, be the zeros of $f$, where we admit repetitions. We next construct by (8) a function $h$ which has zeros at the $a_{n}$ with the multiplicity $n$. Now let $s_{n}=f \cdot\left[f /\left(z-a_{1}\right)\right] \cdots\left[f /\left(z-a_{1}\right) \cdots\left(z-a_{n}\right)\right]$, then $s_{n} \in O\left(\Sigma^{\prime}\right)$ and $h / s_{n}=g_{n} \in O\left(\Sigma^{\prime}\right)$. Since $V\left(z-a_{n}\right)=0$, for otherwise $V=V_{n}, V_{n}$ the valuation for $a_{n}$, we find $V(h)=V\left(s_{n}\right)+V\left(g_{n}\right)$ $=n V(f)+V\left(g_{n}\right)$. This means that $V(h)$ is larger than any positive real number. Consequently $V$ does not exist. 
Combining the preceding observations, we have [5]:

THEOREM 2. The points of $\Sigma^{\prime}$ are in 1-1 correspondence with the closed prime ideals of the ring $O\left(\Sigma^{\prime}\right)$ of analytic functions on $\Sigma^{\prime}$.

The statement of this theorem is closely related to some results dealing with continuous real or complex valued functions on topological spaces $[5,15]$. Suppose that $T$ is a topological space which can be represented as the sum $\mathrm{U}_{n} C_{n}$ of compact sets, $C_{n} \subset C_{n+1}$, such that each compact subset of $T$ is contained in a suitable $C_{n}$. We define in the ring $O^{*}(T)$ of real (or complex) valued continuous functions on $T$ a compact open topology by agreeing that $f_{n} \rightarrow f$ in $O^{*}(T)$ if the sequence $f_{n}$ approaches $f$ uniformly on each compact subset of $T$. Then $O^{*}(T)$ is complete. We have the following lemma.

LeMma 8. Each closed ideal $A$ of $O^{*}(T)$ without zeros on $T$ is equal to $O^{*}(T)$.

Proof. We require of $A$ that there exist for each point $p \in T$ a function $f \in A$ for which $f(p) \neq 0$. Take any $C_{i}$; there exists a point $p_{i j} \in C_{i}$ with a function $f_{i j} \in A$ such that $f_{i j}\left(p_{i j}\right) \neq 0$. By continuity there exists a neighborhood $U_{i j}$ of $p_{i j}$ on which $f_{i j}$ does not vanish. Since $C_{i}$ is compact it can be covered by a finite number $n_{i}$ of such neighborhoods $U_{i j}$ with associated functions $f_{i j} \in A$. Then $f_{i j}^{2}>0$ (or $f_{i j} \bar{f}_{i j}>0$ ) on $U_{i j}$. Let $f_{i}=\sum_{j=1}^{n_{i}} f_{i j}^{2}\left(\sum f_{i j} \bar{f}_{i j}\right)$, then $f_{i}$ does not vanish on $C_{i}$. Let $M_{i}=\max _{p \in C_{i}}\left|f_{i}(p)\right|$. Consider $\sum_{i=1}^{\infty} f_{i} /\left(M_{i} 2^{i}\right)=f$. The sum $f$ is convergent in each $C_{i}$ and hence on each compact subset of $T$. Consequently $f \in O^{*}(T)$ by the completeness of $O^{*}(T)$. Since $A$ is closed and $f_{i} \in A$, we have $f \in A$. By construction $f$ does not vanish on $T$ and therefore $f^{-1} \in O^{*}(T)$, whence $A=O^{*}(T)$.

Let us assume further that $T$ is a completely regular space. We state the following theorem.

THEOREM 3. The closed maximal ideals $M$ of $O^{*}(T)$ are in 1-1 correspondence with the points of $T$.

Proof. If $p$ is a point of $T$ then all $f \in O^{*}(T)$ with $f(p)=0$ form a closed maximal ideal $M_{p}$. Conversely, the functions $f$ of a maximal closed ideal $M$ must have a common zero, $p$, for otherwise $M=O^{*}(T)$ by Lemma 8 . Let $A_{p}$ be the set of all functions vanishing at $p$. Then $M \subseteq A_{p}$ and consequently $M=A_{p}$. The functions of $A_{p}$ cannot vanish at another point $q$ since the complete regularity implies the existence of $f \in A_{p}$ with $f(p)=0, f(q) \neq 0$.

The maximal ideals $M^{*}$ of the ring $O^{*}\left(\Sigma^{\prime}\right)$ of all continuous complex valued functions on $\Sigma^{\prime}$ can be related to the maximal ideals 
$M$ of $O\left(\Sigma^{\prime}\right)$. For each maximal ideal $M$ let the set $M^{*} C O^{*}\left(\Sigma^{\prime}\right)$ consist of all functions $f \in O^{*}\left(\Sigma^{\prime}\right)$ for which there exists an element $m \in M$ such that $f$ vanishes at all zeros of $m$. This set $M^{*}$ is an ideal. By the preceding definition certainly $f g \in M^{*}$ for any $g \in O^{*}\left(\Sigma^{\prime}\right)$. If $f_{1}$ is another function of $M^{*}$ with a corresponding function $m_{1} \in M_{1}$, then $f_{1}+f_{2}$ vanishes at all zeros of a function $h$ in $M$ whose zeros are those common to $m$ and $m_{1}$. (This $h$ may be constructed by the method of Lemma 5.) Furthermore $M^{*}$ is maximal. Suppose $\left(M^{*}, g\right) \subset O^{*}\left(\Sigma^{\prime}\right)$. We pick any $m \neq 0$ in $M$. Then $m$ and $g$ must have zeros in common. For otherwise $k=m \bar{m}+g \bar{g}$ is a function of $\left(M^{*}, g\right)$ without zeros, so that $k^{-1} \in O^{*}\left(\Sigma^{\prime}\right)$ and $1=k^{-1} k \in\left(M^{*}, g\right)$, a contradiction. Let $n$ be an entire function whose zeros are the common zeros of $m$ and $g$. Then $g$ vanishes at all zeros of $n$ and $n \in M$. Hence $g \in M^{*}$.

Conversely each $M^{*}$ determines a prime ideal $M^{*} \cap O\left(\Sigma^{\prime}\right)$ of $O\left(\Sigma^{\prime}\right)$; this ideal is maximal by Lemma 5 . Using the original correspondence $M \rightarrow M^{*}$ we have $M^{*} \cap O\left(\Sigma^{\prime}\right)=M$.

5. The ideal theory in finite extensions of $O\left(\Sigma^{\prime}\right)$. Let $K$ be a finite algebraic extension of degree $n$ over $F\left(\Sigma^{\prime}\right)$. Then each element $k$ of $K$ is the root of an equation

$$
x^{n}+f_{1} x^{n-1}+\cdots+f_{n}=0
$$

whose coefficients are meromorphic functions. Since the valuations $V_{p}$ of $F\left(\Sigma^{\prime}\right)$ are determined by local properties of the functions in $F\left(\Sigma^{\prime}\right)$, we can employ a procedure of the theory of algebraic functions to determine all prolongations $W^{(1)} \cdots W^{(g)}, g=g(p)$, of $V_{p}$ to $K$. We recall that the discrete rank one valuations $W^{(j)}$ can be determined as the unique valuations of the direct summands $K^{(j)}$ of the direct product $K \times F\left(\Sigma^{\prime}\right)_{V}$ where $F\left(\Sigma^{\prime}\right)_{V}$ is the completion of $F\left(\Sigma^{\prime}\right)$ with respect to $V=V_{p}$. Then $1 \leqq g(p) \leqq n$. More precisely $W^{(j)}(k)$, $k \in K$, can be considered as $W^{(1)}\left(k^{S}\right)$ with a suitable conjugate $k^{S}$ of $k$ and a fixed prolongation $W^{(1)}$ of $V_{p}$ [12].

Using the triangle inequality we find:

(16) A function $k \in K$ has at most a denumerable set of zeros and poles, $W_{i}$, in other words valuations $W_{i}$ with $W_{i}(k)=0, \infty$. These valuations $W_{i}$ applied to $F\left(\Sigma^{\prime}\right)$ contract to valuations $V_{i}$ belonging to points $a_{i}$; if $\left\{W_{i}\right\}$ is an infinite set, then $\left|a_{i}\right| \rightarrow \infty . .^{2} \mathrm{~A}$ function $k \in K$ never has an essential singularity at any $W$ of $K$.

We can now construct the Riemann surface $T$ of $K$ and prove, as in the theory of algebraic functions, that the field $F(T)$ of meromorphic functions on $T$ is equal to $K$. Let $O(T)$ be the ring of all

\footnotetext{
${ }^{2} \mathrm{Or},\left\{W_{i}\right\}$ has no infinite subsequence which is compact in $\mathrm{T}$.
} 
entire functions on $T$ and let $R_{W}$ be the valuation ring of $W$ in $K=F(T)$. We shall prove the following generalization of (9),

$$
O(T)=\bigcap_{W} R_{W} .
$$

We first prove [10] the following lemma.

Lemma 9. If $W^{(1)}, \cdots, W^{(\theta)}$ are the prolongations of $V=V_{p}$ to $K$ then $W^{(j)}(k) \geqq 0,1 \leqq j \leqq g$, implies $V\left(f_{i}\right) \geqq 0$ for the coefficients of the defining equation (15). In particular $V(N k)=0$ for the norm $N k$ if all $W^{(j)}(k)$ are 0.

Proof. We imbed $K$ in its least normal extension $L / F\left(\Sigma^{\prime}\right)$ with the Galois group $\{S\}$. Then $\bar{W}^{(v)}(k) \geqq 0$ for all prolongations $\bar{W}^{(v)}$ of $V$ to $L$. Therefore $\prod_{S}\left(x-k^{S}\right)$ has coefficients in the valuation ring $R_{p}$. If $W^{(j)}(k)=0$, then $\bar{W}^{(v)}(k)=0$ and thus $\bar{W}^{(v)}(N k)=0$ or $V(N k)=0$.

As a consequence we note:

$$
W^{(j)}\left[(N k) k^{-1}\right] \geqq 0 \quad \text { if } \quad W^{(j)}(k) \geqq 0 \text { for } 1 \leqq j \leqq g .
$$

Now (17) follows readily. Let $k \in \cap_{W} R_{W}$. Then by Lemma $9, V_{p}\left(f_{i}\right) \geqq 0$ for the coefficients of (15). Consequently $f_{i} \in \bigcap_{p} R_{p}=O\left(\Sigma^{\prime}\right)$ and hence $\bigcap_{W} R_{W} \subseteq O(T)$. The converse inequality is obtained by applying $V$ to (15).

In order to obtain the analogues to Theorems 1 and 2 we use the existence of special local uniformizing variables in $O(T)$ for the prolongations $W^{(1)}, \cdots, W^{(g)}$ of a valuation $V_{p}$.

Lemma 10. There exist elements $t_{1}, \cdots, t_{g}$ in $O(T)$ such that $W^{(i)} t_{i}=1, W^{(j)} t_{i}=0, j \neq i ; 1 \leqq i, j \leqq g$.

Proof. A result of Ostrowski [12] asserts the existence of elements $k_{1}, \cdots, k_{g} \in K$ with $W^{(i)} k_{i}=1, W^{(j)} k_{i}=0, j \neq i, 1 \leqq i, j \leqq g$. Suppose that $W_{\infty}^{(1)}$ is a pole of $k_{i}$ with the conjugates $W_{\infty}^{(2)}, \cdots, W_{\infty}^{(s)}$. Pick $m_{p} \geqq-\min _{1 \leqq \nu \leqq s} W_{\infty}^{(\nu)} k_{i}$. Construct the integers $m_{p}$ for all poles of $k_{i}$ and take, by (8), a function $b_{i} \in O\left(\Sigma^{\prime}\right)$ which has zeros of orders $m_{p}$ at the projections $V_{p}$ of the sets of conjugates $W_{\infty}^{(\nu)}$. Then $b_{i} k_{i}=t_{i}$ has the required properties.

Consequently:

(19) For any distinct rank one valuations $W_{1}, W_{2}$ of $K$ there exist elements $a_{1}, a_{2} \in O(T)$ with $W_{1} a_{1}>0, W_{2} a_{1}=0$ and $W_{1} a_{2}=0, W_{2} a_{2}>0$.

As in §3, equation (10), we define for an ideal $A$ of $K$ with respect to $O(T)$

$$
W_{A}=\left\{\min _{a \in A} W a=W A, \text { all } W\right\} .
$$


Then $W A \neq 0$ for at most a countable infinitude $W$ of valuations, such that $\left|a_{i}\right| \rightarrow \infty$ for the projections $V_{p}$ of the $W_{i}$, if there are infinitely many. Let $\mathscr{W}$ be the additive group of vectors $\left\{m_{W}\right.$, all $\left.W\right\}$ with integers $m_{W}$, such that at most a countable infinitude $m_{W_{i}}$ are distinct from zero, where $\left|a_{i}\right| \rightarrow \infty$ for the corresponding projections to $O\left(\Sigma^{\prime}\right)$.

A direct computation shows that:

$$
\mathscr{U}(A B)=\mathscr{W}(A)+\mathscr{W}(B) \text { for any two ideals } A, B .
$$

Now put all ideals $A, A^{\prime}, \cdots$ with $\mathscr{W} A=W_{A^{\prime}}=\cdots$ in a class $[A]$. As in $\S 3$ the classes $[A]$ form a multiplicative semigroup with $[O(T)]$ for a unit if we define $\mathscr{W}[A]=W A$. More precisely:

THEOREM 4. The classes of ideals $[A]$ form a multiplicative group which is isomorphically mapped in the vector group $W$ by $[A] \rightarrow \mathscr{W}[A]$.

PROof. We may assume without loss of generality that the representative $A$ of $[A]$ lies in $O(T)$. Then the ideal quotient $A^{-1}$ has $W A^{-1}=-W A=-m$ for each $W$. Since $A A^{-1} \subseteq O(T)$ it suffices to exhibit an element $c \in A^{-1}$ with $W c=-m$. Let $t=t_{1}$ be a local uniformizing variable, according to Lemma 10 , such that $W t=W^{(1)} t=1$ and $W^{(2)} t=\cdots=W^{(o)} t=0$ for the conjugates $W^{(2)}, \cdots, W^{(\theta)}$ of $W$. Suppose that $\left\{W_{i}\right\}$ are the valuations with $W_{i} t>0$ and call $V_{i}$ the associated projections on $F\left(\Sigma^{\prime}\right)$. Let $W_{i j}$ be the conjugates of $W_{i}$. Now pick an integer $m_{i} \geqq \max _{j} W_{i j}$. By (8) there exists a function $f \in O\left(\Sigma^{\prime}\right)$ which has zeros at the $V_{i}$ with the multiplicities $m_{i}$. Let $b=t f^{-1}$, then $W(b)=1, W^{(2)}(b)=\cdots=W^{(a)}(b)=0, W_{i j}(b)<0$ for all $i$ and $j$, and finally $W^{\prime}(b)=0$ for all other valuations $W^{\prime}$. Now let $a$ be a nonzero element of $A$, then $W a \geqq m$. Form $a b^{-m}$, then $W\left(a b^{-m}\right)$ $\geqq 0, W^{(v)}\left(a b^{-m}\right)=W^{(v)} a \geqq 0, W_{i j}\left(a b^{-m}\right)=W_{i j} a-m W_{i j} b>0, W^{\prime}\left(a b^{-m}\right)$ $=W^{\prime} a \geqq 0$. Hence $a b^{-m} \in O(T)$ and therefore $c=b^{-m} \in A^{-1}$. Therefore $[A]\left[A^{-1}\right]=[O(T)]$ by $(22)$.

It remains to exhibit for given $\left\{m_{W}\right\}$ an ideal $A$ with $\mathscr{W} A=\left\{m_{W}\right\}$. Let $P_{W}$ be the prime ideal of the valuation $W$ in $K$. We shall exhibit in $A=\bigcap_{W} P_{W}^{m w}$ an element $a$ with $W a=m_{W}$ and $W^{\prime} a \geqq m_{W}$ for all other $W^{\prime}$. By Lemma 10 we can form the product $\prod_{i=1}^{o} t_{i}^{m_{i}}=d \in O(T)$, $m_{i}=m_{W_{i}}$, which has the prescribed orders at $W=W^{(1)}$ and its conjugates $W^{(i)}$. Next we determine in $O\left(\Sigma^{\prime}\right)$ an element $g$ such that $V g=0$ for the projection of $W$ and $V^{\prime} g$ is sufficiently large for the projections of the other $W^{\prime}$ with $m_{W^{\prime}} \neq 0$. Then $a=d g \in A$ and therefore $\mathscr{W}=\left\{m_{\mathbb{W}}\right\}$.

As in (13) any two ideals $A_{1}, A_{2}$ of [ $\left.A\right]$ are related by an equation $A_{1} B_{1}=A_{2} B_{2}$ where $B_{1}, B_{2} \in[O(T)]$. Let $\bar{A}$ be the join of all ideals in 
[A]. Then $\mathscr{W} \bar{A}=\mathscr{W}[A]$ and $\bar{A}=\bigcap_{W} P_{W}^{m_{W}}$ if $\mathscr{W}[A]=\left\{m_{W}\right\}$. Suppose now that we topologize the ring $O(T)$ by using the intrinsic topology of the Riemann surface. Then:

\section{$\bar{A}$ is a closed ideal}

and more precisely the only closed ideal in $[A]$. Using the generalization of Lemma 2 we find that all closed ideals of $K$ with respect to $O(T)$ form a multiplicative group which is isomorphic to the vector group $\mathscr{W}$.

We now turn to the discussion of the prime ideals of $O(T)$ and note first that all prime ideals are maximal as a consequence of Lemma 5. Suppose now that $\mathscr{W} A=\{0\}$ for an ideal $A$ of $O(T)$. We assert:

$$
v\left[A \cap O\left(\Sigma^{\prime}\right)\right]=\{0\} .
$$

For the proof note that for given $V$ with the prolongations $W^{(1)}, \cdots$, $W^{(\theta)}$ there exist elements $a_{1}, \cdots, a_{0}$ in $A$ with

$$
\begin{aligned}
& W^{(1)} a_{1}=0, W^{(2)} a_{1} \geqq 0, \cdots, W^{(o)} a_{1} \geqq 0, \\
& W^{(g)} a_{g} \geqq 0, W^{(2)} a_{g} \geqq 0, \cdots, W^{(\theta)} a_{g}=0 .
\end{aligned}
$$

We apply Lemma 10 and consider the sum, which lies in $A$,

$$
a=a_{1}\left(t_{2} \cdots t_{g}\right)+\cdots+a_{g}\left(t_{1} \cdots t_{g-1}\right) .
$$

Then by construction and the triangle inequality $W^{(1)} a=\ldots$ $=W^{(a)} a=0$. Consequently, by Lemma $9, V(N a)=0$ and $V^{\prime}(N a) \geqq 0$ for all remaining $V^{\prime}$. Thus $N a \in O\left(\Sigma^{\prime}\right)$. Applying (18) we have $(N a) a^{-1} \in O(T)$. Consequently $a\left[(N a) a^{-1}\right]=N a \in A$, whence $V\left(A \cap O\left(\Sigma^{\prime}\right)\right)=0$. Therefore $U\left(A \cap O\left(\Sigma^{\prime}\right)\right)=\{0\}$ since $V$ was chosen arbitrarily.

We notice next that there exist maximal ideals $M$ in $O(T)$ with $\mathscr{W}=\{0\}$. Pick any $B \subset O\left(\Sigma^{\prime}\right)$ with $V B=\{0\}$. Then $B O(T) \subset O(T)$. Hence there exists, by Zorn's Lemma, at least one $M \supset B O(T)$ with the desired properties. These maximal ideals are not closed for otherwise $M \cap O\left(\Sigma^{\prime}\right)$ would be closed and hence equal to $O\left(\Sigma^{\prime}\right)$ by Lemma 1. Because $O(T) / M \supseteq O\left(\Sigma^{\prime}\right) / M \cap O\left(\Sigma^{\prime}\right)$, Lemma 6 implies that $O(T) / M \supset C$ characterizes the nonclosed maximal ideals.

We remark that (23) implies in particular that the intersections $P_{W} \cap O(T)$ are the only closed maximal ideals of $O(T)$. On the other hand the points of the Riemann surface $T$ of $K$ determine the valuations $W$ of $K$ and vice versa. Consequently we have the generalization of Theorem 2: 
The points of $T$ are in 1-1 correspondence with the closed maximal ideals of the ring $O(T)$ of all analytic functions on $T$.

REMARK. In conclusion we point out that the methods of this section apply also to the ideal theory of rings of quasi meromorphic functions [9]. Such functions are meromorphic functions on $\Sigma$ which admit essential singularities at a finite number of points $p_{\infty}, p_{1}, \cdots$, $p_{k}$; in other words, quasi meromorphic functions have locally the character of rational functions on $\Sigma$ with the possible exception of the points $p_{\infty}, p_{1}, \cdots, p_{k}$. To obtain a reduction to the case of the ordinary entire functions suppose that $S$ is an arbitrary (compact) algebraic Riemann surface with the valuations $\left\{W_{S}\right\}$. Pick any $h$ valuations $W_{S, 1}, \cdots, W_{S, h}$. By the Riemann-Roch theorem there exists a function $u$ in the field $F(S)$ of meromorphic functions on $S$ with poles at $W_{S, 1}, \cdots, W_{S, h}$. We consider $F(S)$ as an algebraic extension of $C(u)$. The Riemann surface of $C(u)$ is the complex $u$-sphere and the field of quasi meromorphic functions on $S-\left\{W_{S, 1}, \ldots, W_{S, h}\right\}$ contains $F(S)$ and is an algebraic extension of degree $[F(S): C(u)]$ over the field of meromorphic functions on the $u$-sphere from which the common projection of the $W_{S, \nu}$ is removed. To obtain the ideal theory of the ring of quasi analytic functions on $\Sigma-\left\{p_{\infty}, p_{1}, \cdots, p_{k}\right\}$ we apply the preceding arguments to $F(\Sigma)=C(z) .^{3}$ Finally, the principal ideal theorem (Lemma 3 ) for the closed ideals holds in this case. Similarly Theorem 2 can be extended.

It may be interesting to see which results of the theory of prolongations can be extended to relations between the ideals in the various rings of continuous functions on completely regular spaces and their finitely sheeted covering spaces. It appears that such problems have not been discussed to any extent.

6. The principal ideal theorem. We assume for the moment that $S$ is a compact Riemann surface. Suppose that $\Omega_{v}$ is the ring of all functions in $F(S)$ which are integral over a polynomial ring $C[v]$, $F(S) \supset C[v]$. Then all ideals of $F(S)$ with respect to $\Omega_{v}$ are principal if and only if $S$ has genus 0; this follows immediately from Abel's theorem and Jacobi's inversion theorem [2, 17].

We now show that, in contrast to the preceding compact case, the principal ideal theorem holds for the closed ideals on certain open Riemann surfaces. The existence of integral functions with prescribed zeros depends on a combination of the proof for (8) with the methods leading to Abel's theorem.

\footnotetext{
${ }^{3}$ Quasi analytic functions on $\Sigma-\left\{p_{\infty}, p_{1}, \cdots, p_{k}\right\}$ are quasi meromorphic functions without poles.
} 
We shall assume in the sequel that $K$ is a field of algebroid functions as defined in $\S 5$. Thus, $K$ is the set of all meromorphic functions on a finitely many sheeted covering surface $T$ of $\Sigma^{\prime}$. We agree that the elements of $F\left(\Sigma^{\prime}\right)$ are given as functions of the variable $z$.

Let $O(T)$ be the ring of integral functions over $O\left(\Sigma^{\prime}\right)$ as described in the preceding section. Weierstrass' formula (8) uses the existence of a uniformizing variable $t_{p}$ in $O\left(\Sigma^{\prime}\right)$ for each point $p \in \Sigma^{\prime}$ such that $t_{p}(q) \neq 0$ for all other points $q \in \Sigma^{\prime}$. In the general case we first define a differential $k_{1} d k$ for $k, k_{1} \in K$ as follows [6]:

\section{$k_{1} d k$ is the totality of all local derivatives $k_{1} d k / d t_{W}$}

for all local uniformizing variables $t_{W}$ of $W$ and all valuations $W$ belonging to the points of $T$.

If we use the completions $K_{W}$ of $K$ with respect to $W$ it follows that $W\left(d z / d t_{W}\right)=W(d z)=e-1$ where $e$ is the degree of ramification of $W$ relative to its projection $V$ on $F\left(\Sigma^{\prime}\right)$. In other words, $d z$ is at $W$ the local different of $K_{W} / F\left(\Sigma^{\prime}\right)_{\nu}$. As in the theory of algebraic functions we say that a differential $y d z, y \in K$, has a zero of order $m$ at $W$ if $W(y d z)=m$; a pole of order $m$ is present if $W(y d z)=-m, m>0$. Moreover, the zeros and poles $W_{i}$ of a differential $y d z$ of $K$ have projections $V_{a_{i}}$ with $\left|a_{i}\right| \rightarrow \infty$ if there are infinitely many.

We now construct a differential $d \omega_{W}$ of $K$ which has a logarithmic singularity with the residue +1 at a given valuation $W$. Later $d \omega_{W}$ shall be used to construct the analogue of the prime functions $z-a, a \in C$. Suppose that $a_{1}, \cdots, a_{n}$ are $n$ linearly independent elements of $O(T)$ over $F\left(\Sigma^{\prime}\right)$; we may take $a_{j}=k^{i-1}$ where $k \in O(T)$ is a primitive element of $K / F\left(\Sigma^{\prime}\right)$. Let $a_{j}^{(n)}, 1 \leqq h \leqq n$, be the $n$ conjugates of $a_{j}$. Then the system of linear equations

$$
\sum_{h=1}^{n} b_{i}^{(h)} a_{j}^{(h)}=\delta_{i j}
$$

$\delta_{i j}$ the Kronecker symbols,

has $n$ conjugate sets of solutions $b_{1}^{(n)}, \cdots, b_{n}^{(n)}$, for the independence of the $a_{j}$ 's implies det $\left|a_{j}^{(h)}\right| \neq 0$. Let $b_{i}^{(1)}=b_{i}, 1 \leqq i \leqq n$. We may assume without loss of generality that $z \equiv c \neq 0(\bmod W)$ for the given valuation $W$ of $K$. Since $W$ corresponds to a point of $T$ we agree to denote the value of a function $f \in K$ at $W$ by $f(W) .^{4}$ Consider the differential $d \omega_{W}^{0}=\sum_{i=1}^{n} b_{i}\left[a_{i}(W)\right] d z /(z-c)$. Then $W\left(d \omega_{W}^{0}\right)=-1$ is a consequence of the construction of the $b_{i}$ 's and the remarks on $d z$; we apply the homomorphism determined by $W .^{5}$ The differential $d \omega_{W}^{0}$

${ }^{4}$ In other words, the function-theoretic value $f(W)$ is essentially the residue class of $f$ modulo $W$.

${ }^{5}$ Note $\sum b_{i}(W) a_{i}(W)=1$ and $W(d z)=0$. 
may of course have other singularities. We first remove the potential poles at the conjugates $W^{(h)}$ of $W^{(1)}=W$. Suppose $W^{(h)}\left(d \omega_{W}^{0}\right)=-m_{h}$, $m_{h} \geqq 0$. By Lemma 10, the differential $d \omega_{W}^{0} \prod_{h \rightarrow 1} t_{h}^{m_{h}}=d \omega_{W}^{00}$ has $W^{(h)}\left(d \omega_{h}^{00}\right)$ $=0$ for $h \neq 1$ and $W\left(d \omega_{W}^{00}\right)=-1$. The possible poles $W_{i}$ of $d \omega_{W}^{00}$ form an at most denumerable set with $\left|a_{i}\right| \rightarrow \infty$, if the $V_{a_{i}}$ are the projections of the $W_{i}$. We next pick an integral function $g \in O\left(\Sigma^{\prime}\right)$ which does not vanish at $W$ and its conjugates, that is, $V(g)=0$ for the contraction of $W$ to $F\left(\Sigma^{\prime}\right)$. Moreover, $g$ is to have zeros of sufficiently high orders at the $V_{a_{i}}$. This can be done according to Weierstrass' theorem. Then $W\left(g d \omega_{W}^{00}\right)=-1$ and $W^{\prime}\left(g d \omega_{W}^{00}\right) \geqq 0$ for all other valuations. Finally, we multiply $g d \omega_{W}^{00}$ by a suitable constant and obtain a differential $d \omega_{W}$ with the desired properties.

Now let $I(W)$ be the indefinite integral $\int d \omega_{w}$. Then the exponential $e^{I(T)}$ is a multiplicative function on $T$ which has locally the properties of a meromorphic function on $T .{ }^{6}$ In particular $W\left(e^{I(W)}\right)$ $=-1$ and $W^{\prime}\left(e^{I(W)}\right)=0$ for all other valuations $W^{\prime}$. However, in general, $e^{I(W)}$ will not be a function of $K$. We show next that $e^{I(W)}$ can be normalized so as to lie in $K$ provided a certain hypothesis is valid for the vector space of everywhere finite integrals of $K$.

Let $H^{1}(K)=\left\{d v, W\left(d v / d t_{W}\right) \geqq 0\right.$ for all $\left.W\right\}$ be the vector space of everywhere finite differentials with complex coefficients. Suppose that $H_{1}(K)=\{S, \cdots\}$ is the Betti group of $T$. Then the integrals $\int_{S} d v$ $=(S, d v)$ define for fixed $d v$ a homomorphism of $H_{1}(K)$ into $C$ and dually for fixed $S$ a homomorphism of $H^{1}(K)$ into $C$ since homologous closed curves determine the same definite integral. Let $\phi$ be a homomorphism of $H_{1}(K)$ into $C$. Then it is a well known fact that $\phi$ cannot always be realized as $(S, d v)$ for a suitable $d v$ if integrals on an algebraic compact Riemann surface are considered [2].

We assume the following hypothesis:

(H) Given a homorphism $\phi$ of $H_{1}(K)$ into $C$, then $\phi=(S, d v)$, $S \in H_{1}(K)$ for a suitable differential $d v \in H^{1}(K)$.

Later we shall see that our hypothesis can be proved for certain open Riemann surfaces.

Let $\left\{\pi_{S}\right\}, S \in H_{1}(K)$, be the periods of the normalized integral $\int d \omega_{W}$ which was constructed at the beginning of this section. Then there exists by $(\mathrm{H})$ an everywhere finite differential $d v$ with $(S, d v)=\pi_{S}$ for all $S$. Hence $\int\left(d \omega_{W}-d v\right)=\tau_{W}$ has no periods on $T$ and

$$
e^{\tau W}=t_{W}
$$

is a uniformizing variable for $W$ with $W^{\prime}\left(t_{W}\right)=0$ for all other valuations $W^{\prime}$. It is now easy to show that each closed ideal $A$ of $K$ with

- A multiplicative function $f$ on $T$ reproduces itself but for a constant factor $\mu_{S}$ if it is continued analytically along a closed path $S$ on $T$. 
respect to $O(T)$ is principal, provided $A$ has only a finite number of zeros and poles $W_{i}$ with the multiplicities $m_{i}$. We form the function $a=\prod t_{W_{i}}^{m_{s}}$. Then $W_{a}=W_{A} A$ and therefore $A=a O(T)$. If $A$ has infinitely many zeros and poles then $A$ can still be shown to be principal, but this time the functions $t_{W_{i}}$ have to be modified so that their rate of growth is suitably bounded as $z \rightarrow \infty$; this can be achieved by a combination of the methods in [11] and [13].

We now formulate the following theorem.

THEOREM 5. Let $T$ be the open Riemann surface which is obtained from a compact Riemann surface by omitting a point. Then all closed ideals of meromorphic functions on $T$ with respect to the ring of analytic functions on $T$ are principal. Each closed ideal is uniquely determined by its zeros and poles and their respective multiplicities. The set of zeros and poles of an ideal is at most countable and contains no infinite subsequence which is compact in $T$.

REMARK. The statement of Theorem 5 can be extended to open Riemann surfaces which arise from algebraic Riemann surfaces by omitting more than one point. We note that an analogue to Weierstrass' theorem (8) holds.

We now return to hypothesis $(\mathrm{H})$ and indicate briefly how it can be proved for surfaces $T$ which arise from a compact algebraic Riemann surface $T_{0}$ by omitting a point $\bar{W}, T=T_{0}-\{\bar{W}\}$ [12]. Let $K_{0}$ be the field of algebraic functions of $T_{0}$ and assume that $g$ is the genus. Suppose that $S_{1}, \cdots, S_{v}, T_{1}, \cdots, T_{0}$ is a set of canonical retrosections of $T_{0}$. We may assume, after a suitable deformation of the cuts that $W$ does not lie on any of them. The everywhere finite differentials $d u_{i}$ can now be selected such that, for det $\left|\tau_{k i}\right| \neq 0$,

$$
\left(S_{j}, d u_{i}\right)=\pi(-1)^{1 / 2}, .\left(S_{j}, d u_{i}\right)=0, \quad\left(T_{k}, d u_{i}\right)=\tau_{k i} .
$$

Using the gap theorem of Weierstrass [2], we remark that precisely $g$ integers cannot occur as multiplicities of poles of functions (on $K_{0}$ ) at $\bar{W}$. Since there exist, however, differentials which are poles of any order exclusively at $\bar{W}$, we can pick precisely $g$ differentials $d u_{g+1}, \cdots, d u_{2 \sigma}$ of $K_{0}$ whose multiplicities at $\bar{W}$ are the $g$ missing orders for the functions. Using (28) we can subtract from $d u_{0+1}, \cdots, d u_{2 \sigma}$ suitable linear combinations of the differentials $d u_{1}, \cdots, d u_{o}$ and obtain differentials $d u_{o+1}^{\prime}, \cdots, d u_{2_{g}}^{\prime}$ such that

the multiplicities at $\bar{W}$ are preserved,

$$
\begin{gathered}
\left(S_{h}, d u_{o+j}^{\prime}\right)=0 \\
\left(T_{k}, d u_{o+j}^{\prime}\right)=\tau_{k j .}^{\prime} .
\end{gathered}
$$


The $g$ differentials $d u_{o+b}^{\prime}$ are independent over $C$, for otherwise there would exist a nontrivial linear combination $\int \sum_{j=1}^{g} d u_{0+j}^{\prime} c_{j}=f, c_{j} \in C$, without periods. Hence $f$ would be a function of $K_{0}$ with a forbidden multiplicity at $\bar{W}$. Hence det $\left|\tau_{k j}^{\prime}\right| \neq 0$. We can therefore replace $d u_{0+j}^{\prime}$, $1 \leqq j \leqq g$, by suitable linear combinations $d v_{g+1}, \cdots, d v_{2 g}$ such that

$$
\begin{array}{ll}
\left(S_{h}, d v_{g+j}\right)=0 & \text { for } 1 \leqq h, j \leqq g, \\
\left(T_{k}, d v_{g+j}\right)=\delta_{k j} \pi(-1)^{1 / 2} . &
\end{array}
$$

Finally we add appropriate linear combinations of $d v_{g+1}, \cdots, d v_{2 g}$ to the differentials $d u_{1}, \cdots, d u_{0}$ and obtain $2 g$ independent differentials $d v_{1}, \cdots, d v_{\theta}, d v_{g+1}, \cdots, d v_{2 \sigma}$ with

$$
\begin{aligned}
\left(S_{h}, d v_{i}\right) & =\delta_{h i} \pi(-1)^{1 / 2},\left(T_{k}, d v_{i}\right)=0, & & 1 \leqq h, i, k \leqq g, \\
\left(S_{h}, d v_{g+j}\right) & =0,\left(T_{k}, d v_{g+i}\right)=\delta_{k j} \pi(-1)^{1 / 2}, & & 1 \leqq h, j, k \leqq g .
\end{aligned}
$$

These $2 g$ differentials are now considered on $T=T_{0}-\bar{W}$. Since we do not count $\bar{W}$, it turns out that our differentials are everywhere finite on $T$. We observe that the cuts $S_{i}, T_{i}$ can be placed in a region of $T$ whose projection on $\Sigma^{\prime}$ is given by $|z| \leqq \rho$. Therefore each $S \in H_{1}(K)=H_{1}(T)$ is an integral combination of the $S_{i}, T_{i}$. Suppose then that the homomorphism $\phi$ is given. Then it suffices to know $\phi\left(S_{i}\right), \phi\left(T_{i}\right)$ for then $\phi(S)=\sum n_{i} \phi\left(S_{i}\right)+\sum n_{i}^{\prime} \phi\left(T_{i}\right)$ if $S \sim \sum n_{i} S_{i}$ $+\sum n_{i}^{\prime} T_{i}$. Hence $\phi(S)=(S, d v)$ for a suitable $d v$ as a consequence of (31) and hypothesis $(\mathrm{H})$ is shown to hold.

A further instance of the validity of $(\mathrm{H})$ was recently exhibited by Myrberg [11], who considered $K=F\left(\Sigma^{\prime}\right)\left(f(z)^{1 / 2}\right)$ where $f(z)$ is an entire function with infinitely many zeros. We remark that Myrberg's device of approximating $K$ by hyperelliptic fields can be generalized.

We note in conclusion that the methods used to prove Abel's theorem can be extended to prove a generalized Abel's theorem for meromorphic functions of finite order. The last restriction gives rise to congruences describing the necessary and sufficient conditions for a given set of zeros and poles to belong to a function of finite order [13].

7. Unramified extensions. The function theoretic methods of the preceding section can be further expanded to discuss the unramified finite algebraic extensions $L / K$ in which each valuation $W$ of $K$ has $[L: K]$ distinct prolongations. It can be shown that the unramified abelian extensions are generated by radicals like $(k)^{1 / n}$ where $k$ has zeros and poles on $T$.

More generally, fields of quasi meromorphic functions $\bar{K}$ arising from $T_{0}-\left\{\bar{W}, \bar{W}_{1}, \cdots, \bar{W}_{8}\right\}$ can be treated with our method.

Furthermore, the methods of the theory of algebraic functions 
can be applied directly to prove that a finite group $G$ can be realized as the Galois group of a normal unramified extension $L / K$ if and only if $G$ is a homomorphic image of the Poincaré group $P(\bar{K})=\left\{S_{1}\right.$, $\left.\cdots, S_{g}, T_{1}, \cdots, T_{g}, U_{1}, \cdots, U_{s} ; \prod_{i=1}^{j} S_{i} T_{i} S_{i}^{-1} T_{i}^{-1} \prod_{j=1}^{s} U_{j}=1\right\}$. We note that the roots $\left(t_{j}\right)^{1 / n}$ are unramified functions over $\bar{K}$, where the $t_{j}$ are suitable uniformizing variables for the $\bar{W}_{j}$.

Finally, the analogue of the theory of algebraic correspondences can be established in a variety of cases. It may be worthwhile to compare the results of $\mathrm{H}$. Cartan [4] with the interpretation of meromorphic correspondences by infinite matrices arising in the fields considered by P. J. Myrberg [11].

\section{REFERENCES}

1. A. A. Albert, Modern higher algebra, University of Chicago Press, 1937.

2. G. A. Bliss, Algebraic functions, Amer. Math. Soc. Colloquium Publications, vol. 16, New York, 1933.

3. E. Borel, Leçons sur les fonctions entières, 2d. ed., Paris, 1921.

4. H. Cartan, Sur les variêtés définies par une relation entière, Bull. Sci. Math. vol. 55 (1931) pp. 24-32, 47-64.

5. I. Gelfand, Normierte Ringe, Rec. Math. (Mat. Sbornik) N.S. vol. 51 (1941) pp. 1-23. I. Gelfand and G. E. Silov. Über verschiedene Methoden der Einführung der Topologie in die Menge der maximalen Ideale eines normierten Ringes, Rec. Math. (Mat. Sbornik) N.S. vol. 51 (1941) pp. 25-39. I. Gelfand and A. Kolmogoroff, On rings of continuous functions in topological spaces, C. R. (Doklady) Acad. Sci. URSS. vol. 22 (1939) pp. 11-15.

6. H. Hasse, Theorie der Differentiale in algebraischen Funktionenkörpern mit vollkommenem Konstantenkörpern, J. Reine Angew. Math. vol. 172 (1934) pp. 55-64.

7. O. Helmer, Divisibility properties of integral functions, Duke Math. J. vol. 6 (1940) pp. 345-356.

8. Y. Kawada, Über die Riemannsche Fläche der algebraischen Funktionen, Proc. Imp. Acad. Tokyo vol. 14 (1938) pp. 160-166.

9. E. Maillet, Sur les fonctions entières et quasi entières, Journal de Mathématiques vol. 8 (1902) pp. 329-386.

10. M. Moriya, Bewertungstheoretischer Aufbau der multiplikativen 1dealtheorie, J. Fac. Sci. Imp. Univ. Tokyo. Sect. I vol. 8 (1940) pp. 109-144.

11. P. J. Myrberg, Über analytische Funktionen auf transcendenten Riemannschen Flächen mit reellen Verzweigungspunkten, Acta Math. vol. 76 (1944) pp. 185-224.

12. A. Ostrowski, Untersuchungen zur arithmetischen Theorie der Körper, Math. Zeit. vol. 39 (1935) pp. 269-320.

13. O. Perron, Über transcendente Funktionen auf Riemannschen Flächen, Sitzungsberichte der Heidelberger Akademie der Wissenschaften, 1922-1923, pp. 3-31.

14. S. Stoilow, Leçons sur les principes topologiques de la théorie des fonctions analytiques, Paris, 1938

15. M. H. Stone, Applications of the theory of Boolean rings to general topology, Trans. Amer. Math. Soc. vol. 41 (1937) pp. 375-481.

16. B. L. van der Waerden, Moderne Algebra, 2d ed., Berlin, 1937.

17. H. Weyl, Die Idee der Riemannschen Fläche, 2d ed. Leipzig, 1923. 